\title{
A Randomized Trial Comparing Treatments for Varicose Veins
}

\author{
Julie Brittenden, M.D., Seonaidh C. Cotton, Ph.D., Andrew Elders, M.Sc., \\ Craig R. Ramsay, Ph.D., John Norrie, M.Sc., Jennifer Burr, M.D., \\ Bruce Campbell, M.B., B.S., Paul Bachoo, M.B., Ch.B., \\ Ian Chetter, M.B., Ch.B., M.D., Michael Gough, M.B., Ch.B., \\ Jonothan Earnshaw, D.M., Tim Lees, M.B., Ch.B., M.D., \\ Julian Scott, M.B., Ch.B., M.D., Sara A. Baker, M.Sc., Jill Francis, Ph.D., \\ Emma Tassie, M.Sc., Graham Scotland, Ph.D., Samantha Wileman, Ph.D., \\ and Marion K. Campbell, Ph.D.
}

From the Division of Applied Medicine (J. Brittenden), the Health Services Research Unit (S.C.C., A.E., C.R.R., J.N., G.S., S.W., M.K.C.), and the Health Economics Research Unit (E.T., G.S.), University of Aberdeen, and the Department of Vascular Surgery, NHS Grampian, Aberdeen Royal Infirmary (P.B.), Aberdeen; the School of Medicine, Medical and Biological Sciences, University of St. Andrews, St. Andrews (J. Burr); the Department of Vascular Surgery, Royal Devon and Exeter Hospital, Exeter (B.C.); the Department of Vascular Surgery, Hull Royal Infirmary, Hull (I.C.); the School of Surgery, University of Leeds (M.G.), and Vascular Surgery, St. James University Hospital (J.S.), Leeds; Vascular Surgery, Gloucestershire Royal Hospital, Gloucester (J.E.); Vascular Surgery, Freeman Hospital, Newcastle upon Tyne (T.L.); the Vascular Surgical Unit, Royal Bournemouth Hospital, Bournemouth (S.A.B.); and the School of Health Sciences, City University London, London (J.F.) - all in the United Kingdom. Address reprint requests to Prof. Brittenden at the University of Aberdeen, Division of Applied Medicine, University of Aberdeen, Polwarth Bldg., Foresterhill, Aberdeen AB25 2ZN, United Kingdom, or at j.brittenden@abdn ac.uk.

N Engl J Med 2014;371:1218-27. DOI: 10.1056/NEJMoal400781

Copyright $\odot 2014$ Massachusetts Medical Society.

\section{A BSTRACT}

\section{BACKGROUND}

Ultrasound-guided foam sclerotherapy and endovenous laser ablation are widely used alternatives to surgery for the treatment of varicose veins, but their comparative effectiveness and safety remain uncertain.

\section{METHODS}

In a randomized trial involving 798 participants with primary varicose veins at 11 centers in the United Kingdom, we compared the outcomes of foam, laser, and surgical treatments. Primary outcomes at 6 months were disease-specific quality of life and generic quality of life, as measured on several scales. Secondary outcomes included complications and measures of clinical success.

RESULTS

After adjustment for baseline scores and other covariates, the mean disease-specific quality of life was slightly worse after treatment with foam than after surgery $(\mathrm{P}=0.006)$ but was similar in the laser and surgery groups. There were no significant differences between the surgery group and the foam or the laser group in measures of generic quality of life. The frequency of procedural complications was similar in the foam group (6\%) and the surgery group (7\%) but was lower in the laser group $(1 \%)$ than in the surgery group $(\mathrm{P}<0.001)$; the frequency of serious adverse events (approximately 3\%) was similar among the groups. Measures of clinical success were similar among the groups, but successful ablation of the main trunks of the saphenous vein was less common in the foam group than in the surgery group $(\mathrm{P}<0.001)$.

\section{CONCLUSIONS}

Quality-of-life measures were generally similar among the study groups, with the exception of a slightly worse disease-specific quality of life in the foam group than in the surgery group. All treatments had similar clinical efficacy, but complications were less frequent after laser treatment and ablation rates were lower after foam treatment. (Funded by the Health Technology Assessment Programme of the National Institute for Health Research; Current Controlled Trials number, ISRCTN51995477.) 
LTRASOUND-GUIDED FOAM SCLEROtherapy and thermal ablation techniques such as endovenous laser ablation have become widely used alternatives to surgery for the treatment of varicose veins. Previous randomized trials and meta-analyses have shown these treatments to be effective in terms of short-term technical success and clinician-reported outcomes. ${ }^{1-19}$ Clinical practice guidelines recommend the use of patient-reported quality of life to assess the outcomes of treatment of varicose veins. ${ }^{20}$ Quality of life was a primary outcome measure in two small randomized trials that compared surgery and endovenous laser ablation,,9 but to our knowledge, it has not been assessed as a primary outcome in randomized trials involving foam sclerotherapy.

We performed the Comparison of Laser, Surgery, and Foam Sclerotherapy (CLASS) trial, a large, multicenter, randomized, comparative-effectiveness trial, to assess quality of life and other outcomes of treatment of varicose veins. We compared foam sclerotherapy, laser therapy (with subsequent foam sclerotherapy for residual varicosities, if required), and surgery. ${ }^{21}$

\section{METHODS}

\section{PATIENTS}

We recruited patients requiring treatment of varicose veins in 11 vascular surgery departments in the United Kingdom between November 2008 and October 2012. All patients were assessed by a vascular surgeon and underwent initial duplex ultrasonographic scanning to assess suitability for treatment and entry into the study. Inclusion criteria were an age of 18 years or older, the presence of unilateral or bilateral primary symptomatic varicose veins (grade $\mathrm{C}_{2}$ or higher according to the clinical, etiologic, anatomical, and pathophysiological [CEAP] classification system, with $\mathrm{C}_{0}$ indicating no signs of venous disease, $\mathrm{C}_{1}$ telangiectases or veins $\leq 3 \mathrm{~mm}$ in diameter, $C_{2}$ varicose veins $>3 \mathrm{~mm}$ in diameter, $\mathrm{C}_{3}$ the presence of edema, $\mathrm{C}_{4}$ skin and subcutaneous changes, $\mathrm{C}_{5}$ healed ulcers, and $\mathrm{C}_{6}$ active ulceration, ${ }^{22}$ ), and reflux of the great or small saphenous veins of more than 1 second on duplex ultrasonography. Exclusion criteria were current deep-vein thrombosis, acute superficialvein thrombosis, a diameter of the main truncal saphenous vein of less than $3 \mathrm{~mm}$ or more than $15 \mathrm{~mm}$, tortuous veins considered to be unsuitable for laser treatment, and contraindications to the use of foam or to general or regional anesthesia.

\section{RANDOMIZATION AND STUDY TREATMENT}

A computer-generated randomization system was used and was managed by the Centre for Healthcare Randomised Trials, University of Aberdeen, Aberdeen, United Kingdom. Participants underwent randomization with even assignments to all treatment options available at each investigating center and with stratification according to the number of available options (stratum A, eight hospitals offering all three treatment options; and stratum B, three hospitals offering treatment with only foam or surgery). Treatments were assigned with the use of a minimization algorithm that included center, age ( $<50$ years or $\geq 50$ years), sex, reflux of either the great or the small saphenous veins (or both), and the presence or absence of unilateral or bilateral varicose veins.

Details of treatment methods are described in the published protocol ${ }^{21}$ (available with the full text of this article at NEJM.org). Briefly, surgery consisted of proximal ligation and stripping (of the great saphenous vein only) and concurrent phlebectomies. Foam was produced with the use of the Tessari technique ${ }^{21}$ at a ratio of $0.5 \mathrm{ml}$ of sodium tetradecyl sulfate to $1.5 \mathrm{ml}$ of air (3\% sodium tetradecyl sulfate for saphenous veins and $1 \%$ for varicosities, with a maximum of $12 \mathrm{ml}$ of foam per session). The use of sodium tetradecyl sulfate is licensed, but the trial involved its offlicense use as a foam rather than as its manufactured liquid form. Laser ablation of truncal saphenous veins performed while the patient was under local anesthesia was followed by foam sclerotherapy to residual varicosities at the 6-week follow-up if required, with the exception that one center performed concurrent phlebectomies.

\section{OUTCOME MEASURES}

Outcomes were assessed at baseline and at 6 weeks and 6 months after treatment. The primary outcome measures were patient-reported diseasespecific quality of life, measured with the use of the Aberdeen Varicose Veins Questionnaire (AVVQ), and patient-reported generic (i.e., general) quality of life, measured at 6 months after treatment with the use of the EuroQoL Group 5-Dimension Self-Report Questionnaire (EQ-5D) and the Medical Outcomes Study 36-Item Short-Form Health Survey (SF-36). Another prespecified primary outcome for this trial - 5-year estimated cost-effectiveness, measured as cost per quality-adjusted life-year gained - is not reported here.

The AVVQ is an internationally validated 
change-responsive tool for the assessment of quality of life in patients with varicose veins. ${ }^{20,23-26}$ It consists of 12 questions and a set of mannequin legs on which participants are asked to draw their veins. Scores range from 0 to 100 , with higher scores indicating a worse quality of life. The EQ-5D is a standardized index valuation for health status; it includes five dimensions (mobility, self-care, usual activities, pain or discomfort, and anxiety or depression; scores range from -0.594 to 1.000 , with higher scores indicating a better quality of life) and a visual analogue scale (EQ-5D VAS; scores range from 0 to 100 , with higher scores indicating better health). ${ }^{27}$ The SF-36 is a validated and reliable assessment of quality of life and is widely used for a variety of clinical conditions. ${ }^{28}$ The 36 questions assess eight domains and yield two summary scores (the physical component and the mental component), with each summary score ranging from 0 to 100 and higher scores indicating greater well-being. For all these measures of quality of life, minimal clinically important differences after treatment for varicose veins are not known.

Secondary outcomes were as follows: clinical success at 6 weeks and 6 months, as measured by the proportion of patients with residual varicose veins (assessed by the participant and the nurse), venous clinical severity score (a score composed of nine categories relating to symptoms or signs of venous disease and one category relating to the use of compression; scores range from 0 [no venous disease] to 30 [most severe venous disease]), and complications (assessed by the surgeon or nurse); quality of life according to the AVVQ, EQ-5D, and SF-36 at 6 weeks; the EQ-5D VAS and the eight SF-36 domains at 6 weeks and 6 months; and ablation rates of the main trunks of the saphenous vein according to duplex ultrasonography at 6 weeks and 6 months, assessed with the use of a standardized technique ${ }^{22}$ and reporting tool by independent, accredited vascular technologists (with the exception of one center where scanning was performed by a surgeon who had not performed the treatment). Blinding with respect to the treatment used was not feasible.

\section{STUDY OVERSIGHT}

The trial was approved by the research ethics committee and the Medicines and Healthcare Products Regulatory Authority. Written informed con- sent was obtained from all participants. The trial was overseen by a trial steering committee and an independent data and safety monitoring committee. Data analysis was performed by statisticians at the Centre for Healthcare Randomised Trials. The project management group (the first six and the last four authors) takes responsibility for the accuracy and completeness of the data, analyses, and reporting and for the fidelity of the study to the protocol.

\section{STATISTICAL ANALYSIS}

An intention-to-treat analysis was performed for the prespecified comparisons of treatment with foam versus surgery, involving participants from strata $\mathrm{A}$ and $\mathrm{B}$, and treatment with laser versus surgery, involving participants from stratum A. In addition, we performed a post hoc analysis of laser therapy versus foam sclerotherapy, involving participants from stratum A. The principal analysis of the trial was performed when all participants had completed the 6-month follow-up. Study analyses were conducted according to a prespecified statistical plan (available with the protocol at NEJM.org) with the use of SAS software, version 9.3 (SAS Institute).

To analyze comparisons between groups, we used general linear models with adjustments for covariates used in the minimization algorithm and, where possible, adjustments for baseline scores (AVVQ, EQ-5D, SF-36, and venous clinical severity scores). No adjustment was prespecified for multiple comparisons. However, for the secondary outcome measures presented here, we consider differences to be significant only for $P$ values of less than 0.005 . We analyzed the continuous outcomes with mixed-model repeatedmeasures analysis, with a compound-symmetry covariance matrix and with center fitted as a random effect. Saphenous-vein ablation rates were analyzed with the use of ordinal logistic regression, and rates of complications were analyzed with the use of binary logistic regression. Sensitivity analyses (see Table S1 in the Supplementary Appendix, available at NEJM.org) were carried out in the case of missing AVVQ responses at 6 months. ${ }^{29}$

The initial planned sample size was 1015 patients, which, at a two-sided 5\% significance level, would provide more than $90 \%$ power to detect a difference of 0.25 SD in the AVVQ score for the comparison of foam sclerotherapy with surgery, ${ }^{30,31}$ 
and $80 \%$ power to detect a difference of $0.25 \mathrm{SD}$ in the AVVQ score for the comparison of laser with surgery. The data and safety monitoring committee and trial steering committee approved a revised recruitment target of 779 patients on the basis of data showing that the correlation between the AVVQ score at baseline and at 6 months was better than originally assumed. Only the data and safety monitoring committee was aware of the outcome data according to group assignment during the trial.

\section{RESULTS}

\section{PATIENTS AND TREATMENT}

Of 6592 participants referred from primary care for consideration of treatment, 3369 (51.1\%) met the eligibility criteria, of whom 798 (23.7\%) consented to participation in the trial (Fig. 1) and 785 were included in the trial. The reasons for ineligibility and the reasons that eligible patients declined randomization are summarized in Table S2 in the Supplementary Appendix. Most ineligible participants did not require treatment of the truncal saphenous veins (i.e., the patients were asymptomatic or did not have reflux) or had recurrent varicose veins. Most of the eligible participants who declined to undergo randomization had a preference for a specific treatment. Baseline demographic and clinical data for the three groups are shown in Table 1. Baseline characteristics were generally similar among the groups, with the following exceptions: the diameter of the great saphenous vein was larger in the laser group than in the foam group $(\mathrm{P}=0.008)$, the incidence of reflux below the knee was greater in the foam group than in the laser group $(\mathrm{P}=0.03)$, and the incidence of deep venous reflux was less in the surgery group than in the foam group $(\mathrm{P}=0.006)$. The proportion of treated participants who received their assigned treatment was $97 \%$ in the laser group, $93 \%$ in the foam group, and $87 \%$ in the surgery group. Fully trained staff surgeons performed $73 \%$ of the laser procedures, $77 \%$ of the foam procedures, and $59 \%$ of the surgical procedures; the remaining procedures were performed by surgical trainees. A mean of $83 \mathrm{~J}$ per square centimeter was achieved per laser pulse. Participants who were randomly assigned to treatment with foam or laser had the option of treatment with foam for any residual varicosities at the 6-week follow-up assessment; such treatment was performed in $38 \%$ of participants in the foam group and $31 \%$ of participants in the laser group.

\section{PRIMARY OUTCOMES}

Table 2 summarizes results for the primary outcome measures. At 6 months, the AVVQ score in the foam group was significantly higher (indicating a worse disease-specific quality of life) than that in the surgery group, but the difference was moderate (effect size, $-1.74 ; 95 \%$ confidence interval [CI], -2.97 to $-0.50 ; \mathrm{P}=0.006$ ). The improvement in the AVVQ score in the laser group did not differ significantly from that in the surgery group. There were no significant differences between the groups in the EQ-5D score or the SF-36 physical component score. For the post hoc analysis of treatment with laser versus foam, the only significant difference was in the SF-36 mental component score, which was slightly higher (better generic quality of life) in the laser group than in the foam group (effect size, 1.54; 95\% CI, 0.01 to $3.06 ; \mathrm{P}=0.048$ ).

Results of a post hoc sensitivity analysis of the primary outcomes, which excluded the data from the center where the largest number of participants had been recruited, were generally similar, except that the AVVQ score was significantly worse in the foam group than in the laser group $(\mathrm{P}=0.02)$, and the SF-36 mental component score was worse in the surgery group than in the laser group $(\mathrm{P}=0.04)$ (see Table $\mathrm{S} 3$ in the Supplementary Appendix).

\section{SECONDARY OUTCOMES}

\section{Quality of Life}

Table S4 in the Supplementary Appendix shows results for secondary quality-of-life outcomes. At 6 weeks, significant between-group differences $(\mathrm{P}<0.005)$ included a lower AVVQ score (indicating a better disease-specific quality of life) in the surgery group than in the foam group (effect size, -2.3 ; 95\% CI, -3.7 to -0.9 ) and lower SF-36 scores (indicating a worse generic quality of life) in the surgery group than in the laser group for the domains of bodily pain (effect size, $-2.7 ; 95 \% \mathrm{CI}$, -4.4 to -0.9 ), vitality (effect size, -2.3 ; $95 \% \mathrm{CI}$, -3.9 to -0.8 ), role limitations due to emotional health (effect size, $-2.4 ; 95 \% \mathrm{CI},-4.0$ to -0.8 ), and role limitations due to physical health (effect size, -3.5 ; $95 \%$ CI, -5.2 to -1.8 ). These four SF-36 domain scores did not differ significantly (with $\mathrm{P}<0.005$ considered to indicate statistical signifi- 


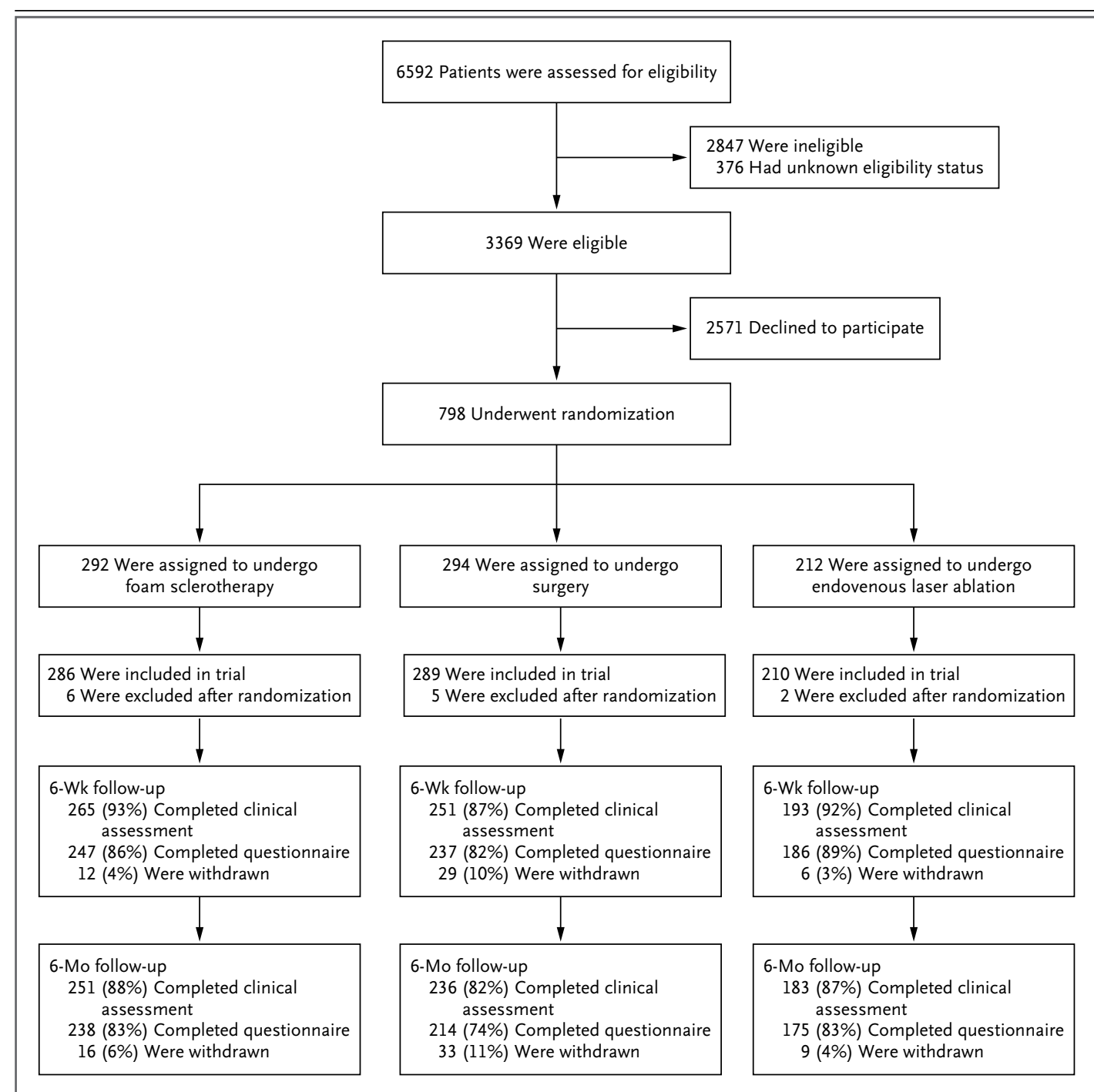

Figure 1. Assessment for Eligibility, Randomization, and Outcomes.

A total of 13 participants (1.6\%) were excluded after randomization because they had veins greater than $15 \mathrm{~mm}$ in diameter (5 participants), recurrent varicose veins ( 3 participants), or coexisting conditions or lack of reflux (5 participants).

cance) between groups at 6 months. For the post hoc comparisons of laser treatment versus foam treatment, only the EQ-5D score was significantly lower (indicating a worse generic quality of life) in the foam group at 6 weeks $(0.044 ; 95 \% \mathrm{CI}$, 0.014 to 0.074$)$.

\section{Clinical Outcomes}

The venous clinical severity score was moderately but significantly lower (indicating less residual venous disease) in the surgery group than the foam group after 6 weeks, but there was no significant difference at 6 months (see Table S5 in the Supplementary Appendix). There was no significant difference in this score between the laser and the surgery groups or between the laser and the foam groups at either time.

At both 6 weeks and 6 months, there were fewer residual varicose veins, as assessed by both participants and nurses, in the surgery group than in the foam group, but the differences were small. There were fewer residual varicose veins in the surgery group than in the laser group at 6 weeks, but not at 6 months, as reported by the nurses but not the participants. There were no significant differences between the laser and the foam 


\begin{tabular}{|c|c|c|c|}
\hline Characteristic & $\begin{array}{l}\text { Laser Group } \\
(\mathrm{N}=210)\end{array}$ & $\begin{array}{l}\text { Foam Group } \\
\qquad(N=286)\end{array}$ & $\begin{array}{l}\text { Surgery Group } \\
\quad(\mathrm{N}=289)\end{array}$ \\
\hline Age $-y r$ & $49.7 \pm 14.4$ & $49.0 \pm 13.3$ & $49.2 \pm 13.7$ \\
\hline Female sex - no. (\%) & $120(57.1)$ & $162(56.6)$ & $163(56.4)$ \\
\hline Body-mass index & $27.0 \pm 4.6$ & $27.1 \pm 4.4$ & $27.7 \pm 4.7$ \\
\hline Unilateral involvement — no. (\%) & $153(72.9)$ & $215(75.2)$ & $196(67.8)$ \\
\hline Previous deep-vein thrombosis — no. (\%) & $6(2.9)$ & $4(1.4)$ & $9(3.1)$ \\
\hline \multicolumn{4}{|l|}{ Saphenous-vein involvement } \\
\hline Great saphenous vein — no. (\%) & $182(86.7)$ & $232(81.1)$ & $239(82.7)$ \\
\hline Widest diameter $-\mathrm{mm} \ddagger$ & $9.1 \pm 3.1$ & $8.4 \pm 2.7$ & $8.7 \pm 2.8$ \\
\hline Reflux above the knee only — no. (\%) & $151(96.2)$ & 169 (93.9) & $180(98.4)$ \\
\hline Reflux above and below the knee - no. (\%)』 & $6(3.8)$ & $11(6.1)$ & $3(1.6)$ \\
\hline Small saphenous vein — no. (\%) & $14(6.7)$ & $21(7.3)$ & $21(7.3)$ \\
\hline Widest diameter - $\mathrm{mm}$ & $7.1 \pm 1.7$ & $7.6 \pm 2.2$ & $7.7 \pm 3.3$ \\
\hline Deep venous reflux — no. (\%) 9 & $28(13.7)$ & $47(16.8)$ & $25(8.9)$ \\
\hline \multicolumn{4}{|l|}{ CEAP classification - no. (\%) } \\
\hline $\mathrm{C}_{2}$, varicose veins $>3 \mathrm{~mm}$ in diameter & $113(54.1)$ & $169(59.1)$ & $147(51.2)$ \\
\hline$C_{3}$, edema & $28(13.4)$ & $35(12.2)$ & $39(13.6)$ \\
\hline $\mathrm{C}_{4}$, skin and subcutaneous changes & $56(26.8)$ & $74(25.9)$ & $90(31.4)$ \\
\hline $\mathrm{C}_{5}$ or $\mathrm{C}_{6}$, skin changes with healed or active venous ulcer & $12(5.7)$ & $8(2.8)$ & $11(3.8)$ \\
\hline \multicolumn{4}{|c|}{$\begin{array}{l}* \text { Plus-minus values are means } \pm \text { SD. CEAP denotes clinical, etiologic, anatomical, and pathophysiological. } \\
\text { The body-mass index is the weight in kilograms divided by the square of the height in meters. } \\
\text { Thor the comparison between the laser and foam groups, } P=0.008 \text {. } \\
\text { I For the comparison between the foam and surgery groups, } P=0.02 \text {. } \\
\text { f For the comparison between the foam and surgery groups, } P=0.006 \text {. }\end{array}$} \\
\hline
\end{tabular}

groups at 6 weeks, but there were fewer residual veins in the laser group than in the foam group at 6 months, as reported by the participants (but not the nurses) (see Table S5 in the Supplementary Appendix).

The frequency of completely successful ablation of great saphenous veins was significantly higher among participants who were randomly assigned to surgery $(84.4 \%)$ or laser treatment $(83.0 \%)$ than among those assigned to foam treatment $(54.6 \%$, $\mathrm{P}<0.001$ for both comparisons). There was no significant difference in success rates between the surgery and the laser groups (Table 3).

\section{Complications}

Serious and nonserious adverse events are shown in Table 4. There were no significant differences between groups in the number of serious adverse events (see Table S6 in the Supplementary Appendix for details of serious adverse events). The frequency of any procedural complications (i.e., complications occurring during treatment) was lower in the laser group $(1.0 \%)$ than in the foam group $(6.2 \%)$ or the surgery group $(7.1 \%)$ ( $\mathrm{P}<0.001$ for both comparisons). At 6 weeks and 6 months, the frequency of overall complications (predominantly lumpiness and skin staining) was greatest in the foam group; the complication rate was significantly greater in the foam group than in the surgery group or the laser group at 6 weeks and was significantly greater in the foam group than in the surgery group at 6 months.

\section{DISCUSSION}

In this multicenter trial comparing foam sclerotherapy or laser treatment with surgery for the treatment of primary varicose veins, quality-oflife measures at 6 months did not differ substantially between groups. However, patients treated with foam had moderately worse outcomes on a 


\begin{tabular}{|c|c|c|c|c|c|c|}
\hline Measure & $\begin{array}{l}\text { Laser } \\
\text { Group } \\
(\mathrm{N}=210)\end{array}$ & $\begin{array}{l}\text { Foam } \\
\text { Group } \\
(\mathrm{N}=\mathbf{2 8 6}) \\
\text { score }\end{array}$ & $\begin{array}{l}\text { Surgery } \\
\text { Group } \\
(N=289)\end{array}$ & $\begin{array}{c}\text { Surgery } \\
\text { vs. Foam'†' }\end{array}$ & $\begin{array}{c}\text { Surgery } \\
\text { vs. Laser: } \\
\text { effect size }(95 \% \mathrm{Cl})\end{array}$ & $\begin{array}{l}\text { Laser } \\
\text { vs. Foam } \int\end{array}$ \\
\hline \multicolumn{7}{|l|}{ AVVQq } \\
\hline Baseline & $17.8 \pm 9.1$ & $17.6 \pm 9.9$ & $18.2 \pm 9.1$ & & & \\
\hline $6 \mathrm{Mo}$ after treatment & $7.9 \pm 8.4$ & $9.1 \pm 7.9$ & $7.8 \pm 7.5$ & $-1.7(-3.0$ to -0.5$)$ & $\|-0.6(-2.2$ to 0.9$)$ & $-1.1(-2.6$ to 0.4$)$ \\
\hline \multicolumn{7}{|l|}{$E Q-5 D * *$} \\
\hline Baseline & $0.792 \pm 0.168$ & $0.803 \pm 0.177$ & $0.784 \pm 0.175$ & & & \\
\hline $6 \mathrm{Mo}$ after treatment & $0.903 \pm 0.171$ & $0.895 \pm 0.174$ & $0.881 \pm 0.202$ & $\begin{array}{c}0.005 \\
(-0.025 \text { to } 0.035)\end{array}$ & $\begin{array}{c}-0.015 \\
(-0.051 \text { to } 0.021)\end{array}$ & $\begin{array}{c}0.025 \\
(-0.010 \text { to } 0.059)\end{array}$ \\
\hline \multicolumn{7}{|c|}{ SF-36 physical component't' } \\
\hline Baseline & $48.6 \pm 7.8$ & $48.9 \pm 8.0$ & $48.2 \pm 8.6$ & & & \\
\hline $6 \mathrm{Mo}$ after treatment & $52.6 \pm 7.3$ & $52.3 \pm 8.5$ & $52.4 \pm 8.9$ & $1.0(-0.2$ to 2.3$)$ & $0.1(-1.4$ to 1.6$)$ & $0.7(-0.8$ to 2.1$)$ \\
\hline \multicolumn{7}{|l|}{ SF-36 mental componentit' } \\
\hline Baseline & $51.9 \pm 9.0$ & $52.4 \pm 8.7$ & $51.2 \pm 9.4$ & & & \\
\hline $6 \mathrm{Mo}$ after treatment & $53.5 \pm 7.7$ & $52.2 \pm 9.1$ & $52.1 \pm 8.6$ & $0.2(-1.1$ to 1.6$)$ & $-1.3(-2.9$ to 0.2$)$ & 1.5 (0.0 to 3.1$)$ \\
\hline \multicolumn{7}{|c|}{ 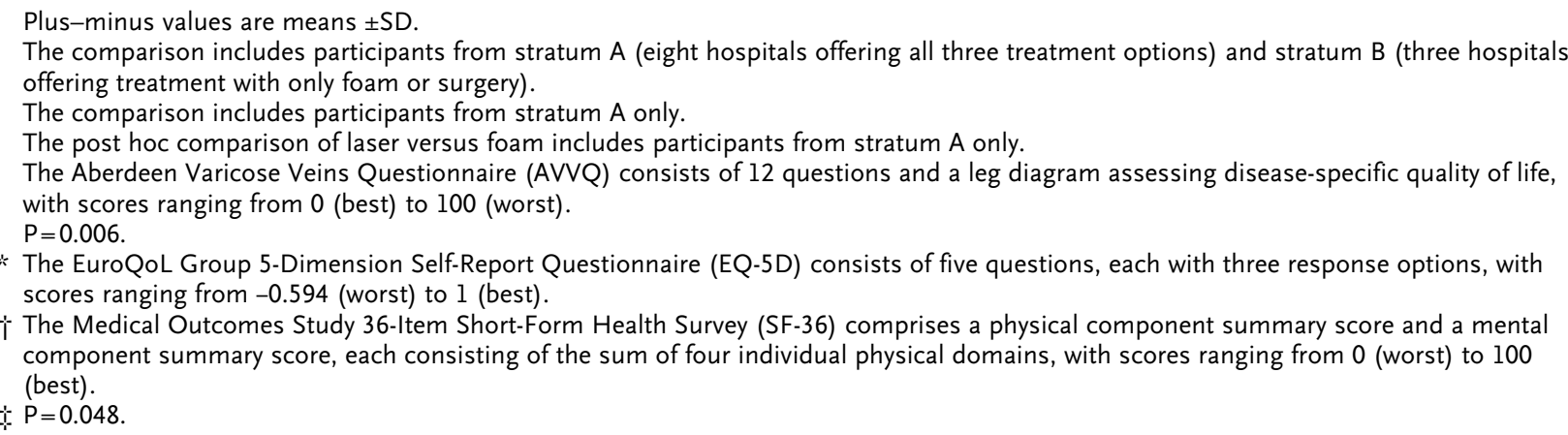 } \\
\hline
\end{tabular}

measure of disease-specific quality of life (AVVQ) than did those who underwent surgery. These differences were small, and their clinical importance is uncertain.

The frequency of complications (e.g., lumpiness, skin staining, and numbness) was lower after treatment with laser than after treatment with either foam or surgery; these differences are likely to have affected quality of life. However, the use of foam sclerotherapy for residual varicosities in a third of the participants in the laser group at 6 weeks may have attenuated early quality-of-life benefits associated with this treatment as compared with treatment with foam or surgery. ${ }^{31}$

The baseline scores and overall improvements in quality of life that we observed were similar to those reported in smaller European random- ized trials, supporting the generalizability of our findings. . $, 5,68-10,12,32$ The nature and frequency of complications were also similar to those previously reported in the literature. ${ }^{1-16}$

The three groups had similar improvements in the venous clinical severity score at 6 months. However, successful ablation of the great saphenous veins at 6 weeks occurred significantly less often after foam treatment (complete ablation, $55 \%$; partial ablation with a patent segment and no reflux, 23\%) than after either surgery (complete ablation, $84 \%$; partial ablation, $6 \%$ ) or laser treatment (complete ablation, 83\%; partial ablation, $8 \%$ ). Observed ablation rates, in particular for foam sclerotherapy, were lower than the rates in some previous studies, which used less strict definitions of success, ${ }^{1,6,10,14}$ but were similar to the rates reported in two randomized trials that 


\begin{tabular}{|c|c|c|c|c|c|c|}
\hline Variable & $\begin{array}{l}\text { Laser } \\
\text { Group }\end{array}$ & $\begin{array}{l}\text { Foam } \\
\text { Group }\end{array}$ & $\begin{array}{l}\text { Surgery } \\
\text { Group }\end{array}$ & $\begin{array}{c}\text { Surgery } \\
\text { vs. Foam'广 }\end{array}$ & $\begin{array}{c}\text { Surgery } \\
\text { vs. Laser:t: } \\
\text { odds ratio }(95 \%\end{array}$ & $\begin{array}{l}\text { Laser } \\
\text { vs. Foam } \int\end{array}$ \\
\hline \multicolumn{7}{|l|}{ At $6 \mathrm{wk}$} \\
\hline No. of patients & 153 & 205 & 192 & & & \\
\hline Complete success — no. (\%) & $127(83.0)$ & $112(54.6)$ & $162(84.4)$ & & & \\
\hline Partial success without reflux - no. (\%) & $13(8.5)$ & $47(22.9)$ & $12(6.3)$ & & & \\
\hline Partial success with reflux - no. (\%) & $10(6.5)$ & $9(4.4)$ & $9(4.7)$ & & & \\
\hline Failure - no. (\%) & $3(2.0)$ & $37(18.0)$ & $9(4.7)$ & $5.1(3.1-8.5) 9$ & $1.2(0.6-2.3)$ & $3.1(1.8-5.4) 9$ \\
\hline \multicolumn{7}{|l|}{ At 6 mo } \\
\hline No. of patients & 141 & 182 & 173 & & & \\
\hline Complete success — no. (\%) & $116(82.3)$ & $79(43.4)$ & $135(78.0)$ & & & \\
\hline Partial success without reflux - no. (\%) & $13(9.2)$ & $35(19.2)$ & $4(2.3)$ & & & \\
\hline Partial success with reflux — no. (\%) & $3(2.1)$ & $9(4.9)$ & $20(11.6)$ & & & \\
\hline Failure - no. (\%) & $9(6.4)$ & $59(32.4)$ & $14(8.1)$ & $4.9(3.1-7.9) 9$ & $0.8(0.4-1.5)$ & $4.8(2.8-8.5) 9$ \\
\hline \multicolumn{7}{|c|}{$\begin{array}{l}\text { - Complete success was defined as complete occlusion at the treated vein, partial success as a patent segment of treated vein with or withou } \\
\text { reflux, and failure as more than one patent segment. } \\
t \text { The comparison includes participants from strata A and B. } \\
\text { The comparison includes participants from stratum A only. } \\
\text { व The post hoc comparison of laser versus foam includes participants from stratum A only. } \\
\qquad P \leq 0.01 \text {. }\end{array}$} \\
\hline
\end{tabular}

used definitions of technical success that were similar to ours. ${ }^{12,15}$ In contrast to the assessment of ablation in previous trials, we determined whether ablation was complete or partial on the basis of duplex ultrasound scans obtained by independent, accredited vascular technologists rather than by the surgeons who had performed the treatment. The disparity between clinical measures of success and technical success observed in this study has also been observed in other trials of treatment for varicose veins. ${ }^{18,19}$

For patients undergoing laser treatment, concurrent treatment of varicosities remains controversial. ${ }^{16} \mathrm{~A}$ previous, single-center trial showed significant improvements in the AVVQ score at 6 weeks among patients who underwent phlebectomy concomitantly with laser therapy, as compared with those who underwent laser therapy alone. ${ }^{33}$ In our study, participants in the laser group received treatment directed to the main saphenous vein, without concomitant phlebectomies (except at one center). We found no significant differences in the AVVQ score among patients undergoing laser treatment versus surgery at 6 weeks, despite the use of concomitant phlebectomies in the surgery group.
The limitations of our study should be acknowledged. First, it was not feasible for the participants or assessors to be kept unaware of the assigned treatment. Second, we did not include a group of patients assigned to a sham procedure and thus cannot assess the effect of treatment relative to such a control. Third, eight comparisons involved primary outcomes, and a large number of comparisons involved secondary outcomes; thus, it is likely that some differences may have occurred by chance. We considered differences for secondary outcome measures to be significant only for P values of less than 0.005 . Fourth, longer follow-up is needed to assess the durability of effects. Finally, although radiofrequency ablation is also used to treat varicose veins, we did not include this method in our trial; other studies have suggested that it results in clinical outcomes similar to those associated with laser treatment. ${ }^{16}$

In conclusion, our multicenter trial comparing the clinical effectiveness of endovenous laser ablation, foam sclerotherapy, and surgery for the treatment of varicose veins showed no clinically substantial between-group differences in quality of life. Moderate differences in disease-specific quality of life favored surgery over treatment 


\begin{tabular}{|c|c|c|c|}
\hline \multirow[t]{2}{*}{ Event } & Laser Group & Foam Group & Surgery Group \\
\hline & \multicolumn{3}{|c|}{ number/total number (percent) } \\
\hline Serious adverse events overall & $7 / 210(3.3)$ & $11 / 286(3.8)$ & $10 / 289(3.5)$ \\
\hline Serious adverse events related to treatment & $2 / 210(1.0)$ & $3 / 286(1.0)$ & $4 / 289(1.4)$ \\
\hline \multicolumn{4}{|l|}{ Other complications } \\
\hline Any procedural complication during treatment & $2 / 205(1.0)$ & $17 / 275(6.2) \dagger$ & $19 / 267(7.1) \dagger$ \\
\hline Any complication at $6 \mathrm{wk}$ & $103 / 193(53.4)$ & $219 / 265(82.6) \dagger$ & $168 / 251(66.9) \div$ \\
\hline Any complication at $6 \mathrm{mo}$ & $89 / 183(48.6)$ & $144 / 251(57.4) \sqrt{ }$ & $109 / 236(46.2)$ \\
\hline Numbness at 6 wk & $22 / 193(11.4) 9$ & $15 / 265(5.7)$ & $45 / 251(17.9) \|$ \\
\hline Numbness at 6 mo & $17 / 183(9.2) 9$ & $10 / 251(4.0)$ & $37 / 236(15.6) \|$ \\
\hline Persistent bruising at $6 \mathrm{wk}$ & $10 / 193(5.2)$ & $49 / 265(18.5) \dagger$ & $32 / 251(12.7) \ddagger$ \\
\hline Persistent bruising at 6 mo & $25 / 183(13.6)$ & $38 / 251(15.2)$ & $40 / 236(17.0)$ \\
\hline Persistent tenderness and discomfort at $6 \mathrm{wk}$ & $41 / 193(21.2)$ & $122 / 265(46.0) \dagger$ & $79 / 251(31.5) \dagger 9$ \\
\hline Skin loss or ulceration at $6 \mathrm{wk}$ & $0 / 193$ & $2 / 265(0.8)$ & $1 / 251(0.4)$ \\
\hline Skin loss or ulceration at $6 \mathrm{mo}$ & $1 / 183(0.6)$ & $2 / 251(0.8)$ & $0 / 236$ \\
\hline Lumpiness at $6 \mathrm{wk}$ & $36 / 193$ (18.7) & $171 / 265(64.5) \dagger$ & $83 / 251(33.1) \ddagger$ \\
\hline Lumpiness at $6 \mathrm{mo}$ & $25 / 183(13.6) \mathbb{\int}$ & $67 / 251(26.6) \div * *$ & $17 / 236(7.2) \div$ \\
\hline Development of thread vein at $6 \mathrm{wk}$ & $10 / 193(5.2)$ & $27 / 265(10.2) \div$ & $21 / 251(8.4)$ \\
\hline Development of thread vein at 6 mo & $24 / 183(13.2)$ & $34 / 251(13.6)$ & $26 / 236(11.0)$ \\
\hline Skin staining at $6 \mathrm{wk}$ & $18 / 193(9.3)$ & $105 / 265(39.6) \dagger^{\prime}$ & $20 / 251(8.0)$ \\
\hline Skin staining at $6 \mathrm{mo}$ & $32 / 183(17.4) \mathbb{\int}$ & $92 / 251(36.6) \dagger * * *$ & $24 / 236(10.2)$ \\
\hline
\end{tabular}

* The comparison between the foam group and the surgery group includes participants from strata A and B; the comparison between the surgery group and the laser group includes participants from stratum $A$ only. The post hoc analysis of the laser group versus the foam group includes participants from stratum A only; $31 \%$ of the patients in the laser group also underwent foam treatment of varicosities. Thread veins are small clusters of blue or red veins.

$\dagger \mathrm{P}<0.001$ for the comparison with the laser group.

$P \quad P<0.05$ for the comparison with the laser group.

$\int \mathrm{P}<0.05$ for the comparison with the surgery group.

q $\mathrm{P}<0.05$ for the comparison with the foam group.

$\mathrm{P}<0.001$ for the comparison with the foam group.

$* * \mathrm{P}<0.001$ for the comparison with the surgery group.

with foam, and moderate differences in generic quality of life favored laser treatment over foam. All treatments had similar clinical efficacy, but there were fewer complications after laser treatment, and ablation rates were lower after treatment with foam.

The views expressed in this article are those of the authors and do not necessarily reflect the views of the National Institute for Health Research, the National Health Service, the Department of Health, or the funders that provided institutional support for this study.

Supported by a grant from the Health Technology Assessment Programme of the National Institute for Health Research $(06 / 45 / 02)$. The Health Services Research Unit is funded by the Chief Scientist Office of the Scottish Government Health Directorate.
Disclosure forms provided by the authors are available with the full text of this article at NEJM.org.

We thank Janice Cruden for her secretarial support and data management; Gladys McPherson and the programming team at the Centre for Healthcare Randomised Trials; Tracey Davidson, Lynda Constable, Jackie Ellington, Laura Elliott, and Yvonne Fernie for help with scoring the Aberdeen Varicose Vein Questionnaire; Luke Vale and Laura Ternent, our original economists in the group; members of the Project Management Group for their ongoing advice and support of the trial; members of the study team (Graeme MacLennan, Maria Prior, and Denise Bolsover) who contributed to the behavioral recovery component of the trial; the independent members of the trial steering committee (Alun Davies [chair], Ian Loftus, and Jane Nixon) and the data and safety monitoring committee (Gerry Stansby [chair], Winston Banya, and Marcus Flather); and the staff members at recruitment sites (see the Supplementary Appendix) who facilitated recruitment, treatment, and follow-up of trial participants. 
REFERENCES

1. Biemans AAM, Kockaert M, Akkersdijk GP, et al. Comparing endovenous laser ablation, foam sclerotherapy, and conventional surgery for great saphenous varicose veins. J Vasc Surg 2013;58:727-34. 2. Samuel N, Carradice D, Wallace T, Mekako A, Hatfield J, Chetter I. Randomized clinical trial of endovenous laser ablation versus conventional surgery fo small saphenous varicose veins. Ann Surg 2013;257:419-26.

3. Rass $\mathrm{K}$, Frings $\mathrm{N}$, Glowacki $\mathrm{P}$, et al. Comparable effectiveness of endovenous laser ablation and high ligation with stripping of the great saphenous vein: twoyear results of a randomized clinical trial (RELACS study). Arch Dermatol 2012;148: 49-58.

4. Flessenkämper I, Hartmann M, Stenge D, Roll S. Endovenous laser ablation with and without high ligation compared with high ligation and stripping in the treatment of great saphenous varicose veins: initial results of a multicentre randomized controlled trial. Phlebology 2013;28. 16-23.

5. Carradice D, Mekako AI, Mazari FAK Samuel N, Hatfield J, Chetter IC. Randomized clinical trial of endovenous laser ablation compared with conventional sur gery for great saphenous varicose veins. Br J Surg 2011;98:501-10.

6. Rasmussen LH, Lawaetz M, Bjoern L, Vennits B, Blemings A, Eklof B. Randomized clinical trial comparing endovenous laser ablation, radiofrequency ablation, foam sclerotherapy and surgical stripping for great saphenous varicose veins. $\mathrm{Br} J$ Surg 2011;98:1079-87.

7. Pronk P, Gauw SA, Mooij MC, et al. Randomised controlled trial comparing sapheno-femoral ligation and stripping of the great saphenous vein with endovenous laser ablation (980 nm) using loca tumescent anaesthesia: one year results. Eur J Vasc Endovasc Surg 2010;40:649-56. 8. Christenson JT, Gueddi S, Gemayel G, Bounameaux $\mathrm{H}$. Prospective randomized trial comparing endovenous laser ablation and surgery for treatment of primary great saphenous varicose veins with a 2-year follow-up. J Vasc Surg 2010;52:1234-41.

9. Darwood RJ, Theivacumar N, Dellagrammaticas D, Mavor AI, Gough MJ. Randomized clinical trial comparing endovenous laser ablation with surgery for the treatment of primary great saphenous varicose veins. Br J Surg 2008;95:294-301. 10. Rasmussen LH, Bjoern L, Lawaetz M, Blemings A, Lawaetz B, Eklof B. Randomized trial comparing endovenous laser ablation of the great saphenous vein with high ligation and stripping in patients with varicose veins: short-term results. J Vasc Surg 2007;46:308-15.

11. Rasmussen LH, Bjoern L, Lawaetz M, Lawaetz B, Blemings A, Eklöf B. Randomised clinical trial comparing endovenous laser ablation with stripping of the great saphenous vein: clinical outcome and recurrence after 2 years. Eur J Vasc Endovasc Surg 2010;39:630-5.

12. Lattimer CR, Azzam M, Kalodiki E, Shawish E, Trueman P, Geroulakos G. Cost and effectiveness of laser with phlebectomies compared with foam sclerotherapy in superficial venous insufficiency: early results of a randomised controlled trial. Eur J Vasc Endovasc Surg 2012;43: 594-600.

13. Shadid N, Ceulen R, Nelemans P, et al. Randomized clinical trial of ultrasound-guided foam sclerotherapy versus surgery for the incompetent great saphenous vein. Br J Surg 2012;99:1062-70.

14. Figueiredo M, Araújo S, Barros NJ Jr, Miranda FJ Jr. Results of surgical treatment compared with ultrasound-guided foam sclerotherapy in patients with varicose veins: a prospective randomised study. Eur J Vasc Endovasc Surg 2009;38:758-63. 15. Wright D, Gobin JP, Bradbury A, et al. Varisolve polidocanol microfoam compared with surgery or sclerotherapy in the management of varicose veins in the presence of trunk vein incompetence: European randomised controlled trial. Phlebology 2006;21:180-90.

16. Varicose veins in the legs CG168: the diagnosis and management of varicose veins. London: National Institute for Health and Care Excellence (http:/guidance.nice .org.uk/CG168).

17. van den Bos R, Arends L, Kockaert M, Neumann M, Nijsten T. Endovenous therapies of lower extremity varicosities: a metaanalysis. J Vasc Surg 2009;49:230-9.

18. Luebke T, Brunkwall J. Systematic review and meta-analysis of endovenous radiofrequency obliteration, endovenous laser therapy, and foam sclerotherapy for primary varicosis. J Cardiovasc Surg (Torino) 2008;49:213-33.

19. Nesbitt C, Eifell RK, Coyne P, Badri H, Bhattacharya V, Stansby G. Endovenous ablation (radiofrequency and laser) and foam sclerotherapy versus conventional surgery for great saphenous vein varices. Cochrane Database Syst Rev 2011;10:CD005624. 20. Gloviczki P, Comerota AJ, Dalsing $\mathrm{MC}$, et al. The care of patients with varicose veins and associated chronic venous diseases: clinical practice guidelines of the Society for Vascular Surgery and the American Venous Forum. J Vasc Surg 2011; 53:Suppl:2S-48S.
21. The CLASS Study: Comparison of LAser, Surgery and foam Sclerotherapy as a treatment for varicose veins (ISRCTN 51995477; EudraCT 2008-001069-26). Lancet 2010;Lancet protocol 09PRT/2275 (http://www.thelancet.com/protocol -reviews/09PRT-2275).

22. Kundu S, Lurie F, Millward SF, et al. Recommended reporting standards for endovenous ablation for the treatment of venous insufficiency: joint statement of The American Venous Forum and The Society of Interventional Radiology. J Vasc Surg 2007;46:582-9.

23. Garratt AM, Macdonald LM, Ruta DA, Russell IT, Buckingham JK, Krukowski $\mathrm{ZH}$. Towards measurement of outcome for patients with varicose veins. Qual Health Care 1993;2:5-10.

24. Smith JJ, Garratt AM, Guest $M$, Greenhalgh RM, Davies AH. Evaluating and improving health-related quality of life in patients with varicose veins. J Vasc Surg 1999;30:710-9.

25. Garratt AM, Ruta DA, Abdalla MI, Russell IT. Responsiveness of the SF-36 and a condition-specific measure of health for patients with varicose veins. Qual Life Res 1996;5:223-34

26. Klem TMAL, Sybrandy JEM, Wittens CHA, Essink Bot ML. Reliability and validity of the Dutch translated Aberdeen Varicose Vein Questionnaire. Eur J Vasc Endovasc Surg 2009;37:232-8.

27. EQ-5D. Rotterdam, the Netherlands: EuroQol (http://www.euroqol.org).

28. Ware JE Jr. SF-36 Health Survey update (http://www.sf-36.org/tools/sf36.shtml).

29. White IR, Horton NJ, Carpenter J, Pocock SJ. Strategy for intention to treat analysis in randomised trials with missing outcome data. BMJ 2011;342:d40.

30. Ratcliffe J, Brazier JE, Campbell WB, Palfreyman S, MacIntyre JB, Michaels JA. Cost-effectiveness analysis of surgery versus conservative treatment for uncomplicated varicose veins in a randomized clinical trial. Br J Surg 2006;93:182-6.

31. Cohen J. Statistical power analysis for the behavioral sciences. 2nd ed. Hillsdale, NJ: Lawrence Erlbaum Associates, 1988.

32. PROMs outcomes summary by provider, April 2010-March 2011 (http://data.gov .uk/dataset/provisional-monthly-patient -reported-outcome-measures-proms-in -england-april-2010-to-march-2011).

33. Carradice D, Mekako AI, Hatfield J, Chetter IC. Randomized clinical trial of concomitant or sequential phlebectomy after endovenous laser therapy for varicose veins. Br J Surg 2009;96:369-75.

Copyright (C) 2014 Massachusetts Medical Society. 